\title{
A Sustainable Approach for Smart Supply Chain with Quality Humiliation and Carbon Emission Cost
}

\author{
P.Mala ${ }^{1}$, S.Priyan ${ }^{2}$ \\ ${ }^{1}$ Department of Mathematics, Kamaraj College of Engineering \& Technology \\ Virudhunaga, Tamilnadu, India. \\ Email: gurupmala [AT] gmail.com \\ ${ }^{2}$ Department of Mathematics, Hindustan Institute of Technology and Science (Deemed to be University) \\ Chennai, Tamil Nadu, India \\ Email: jaisilpriyan [AT] gmail.com
}

\begin{abstract}
Most decisive principles affecting customers' ordering decision is quality of the products. The intention of the work is to provide the sustainable decisions such as order lot-size and total number of lot-size to create a smart SC in view of minimum carbon emissions and joint total cost. This research considers an imperfect production vendor-buyer inventory system involving quality degradation of raw material and carbon emission cost. The vendor's production process produces some imperfect products, even manufactured products inspect twice, which are examined by buyer and those are return back to vendor in the subsequent shipment. We incorporate the emissions throughout the production processes, transport and storage. Mathematical based inventory model is developed to attain the sustainable decisions which are illustrated numerically.
\end{abstract}

Keywords - Quality humiliation, two stage inspection, carbon emission, optimization

\section{INTRODUCTION}

Today's crucial challenges around the world are mobbing and contamination as a result of increasing ecommerce and parcel deliveries in cities. Cities worldwide are expected to standardize delivery traffic in and out of metro cities. We need smart SC to meet these challenges. It provides a wide range of beneficial information so as to support the growth of urban freight transportation tactics. Collaboration between organizations is the system of pooling knowledge, resources and relationships for the sake of pursuing shared pursuits. In these days' notably traumatic enterprise surroundings, collaborative inventory system successfully provides buying and selling partners with realtime business information that could otherwise be unavailable or not to be had rapid sufficient. Hence, supply chain (SC) cooperation has been the theme of a huge deal of discuss in the literature for several decades (ref [1], [2]).

Raw-materials, including ingredients, processing aids, and wrapping, are the base of finished items. In a SC, all activities from delivery of raw-materials to finished items must be included. Today one of the greatest challenges facing manufacturers is the irregularity of raw-material qualities. Quality variation of the raw-materials used in manufacturing will often lead to dissimilarity in the quality of the final items (i.e. imperfect finished product). In spite of raw material quality degradation and imperfect production process being extremely happen in industries no paper diagnosed these issues concurrently. So it is significant to comprehend what is important for raw-material quality, and how we can influence these factors, if we are to attain a consistent and right quality of items. In this paper we use two stage inspections. Darwish and Ben-Daya [3] studied effects on inspection error and prevention of two stage production. Chung [4] investigated flawed process and demand effects on inspection scheduling and supply chain replenishment strategy. Degradation products are unwanted chemicals that can develop during the manufacturing, transportation, and storage of products and can affect the efficacy of products. Here we use quality degradation for raw materials. Mantin and Jiang [5] presented strategic inventories with quality degradation. Priyan and Uthayakumar [6] designed a mathematical modeling and computational algorithm to solve multi-echelon multi-constraint inventory problem with errors in quality inspection. Fauza et al. [7] developed inventory strategy for food items involving quality degradation. Priyan and Mala [8] used a game theory approach for optimal inventory system for pharmaceutical products incorporating quality degradation with expiration date. 
The common practice in the international market is the integrated inventory management system, and it provides economic benefits both to the seller and the buyer. In recent times, seller and buyer integration have been a substantial area of focus by most integrated inventory management systems. In general, the entire production-inventory system emits carbon in every stage. The environmental degradation is high nowadays due to excessive carbon emission. Numerous countries are under rising pressure to hold back carbon emissions with a few precise aims to reduce emissions or even law enactment preparation to control emissions. Li et al. [9] incorporated carbon emissions into a production and transportation operation problem and developed two optimization models for a multi-echelon supply chain system. Zhengyi et al. [10] presented inventory management for one warehouse multi retailer system with carbon emission cost.

The remainder of the manuscript follows the subsequent structure: section 2 indicates notations and assumptions. In section 3 , the mathematical formulations of the inventory model is constructed and procedure for solution is indicated in section 4. Section 5 contains the discussion for analysis for sensitivity and numerical. Section 6 discusses the summary of the manuscript.

\section{NOTATION AND ASSUMPTIONS}

\subsection{Notations}

$\mathrm{T} \quad$ manufacturing cycle time

$\mathrm{P} \quad$ production rate of perfect item

$Q \quad$ delivery quantity of perfect goods to buyer

M amount of raw-materials for a production cycle

D demand rate (units/unit time)

$p_{\text {raw }} \quad$ purchase cost of raw material

$Z_{\text {raw }} \quad$ delivery cost of raw-material

$C_{\text {loss }} \quad$ cost of quality loss for raw material

$H_{\text {raw }}$ raw-material's holding cost

$\mathrm{k}$ deterioration rate

Q0 ordering lot-size (units)

Q perfect finished goods to sell in a cycle time $\mathrm{T}$

$\mathrm{H}_{1} \quad$ holding cost for vendor (perfect item)

$\mathrm{P}_{0} \quad$ production rate

$\mathrm{P} \quad$ production rate of perfect item

$n \quad$ number of deliveries in the whole planning horizon

$\mathrm{S} \quad$ set up cost

$\mathrm{C}_{0} \quad$ inspection cost per units

$\mathrm{C}_{1} \quad$ rework cost per units

$\mathrm{C}_{2} \quad$ disposal cost per units

$c_{e} \quad$ carbon emission cost per unit

$\mathrm{Q}_{0} \quad$ raw material ordering quantity (units)

$\alpha \quad$ rate of proportion of imperfect products in the start of production

$\beta \quad$ rate of proportion of perfect products in the rework items

$\mathrm{A}_{1} \quad$ buyer's ordering cost

$\mathrm{H}_{2} \quad$ holding cost of buyer

$\mathrm{H}_{3} \quad$ buyer's handling cost

c transportation cost

$Q_{R}^{\max } \quad$ extreme quality level of raw-material

$Q_{R}^{\min } \quad$ lowest quality level of raw-material

$Q_{R}(t)$ residual quality level of raw-material at time $t$

\subsection{Assumptions}

1. The vendor's raw-material is one time order and its extreme quality level $\left(Q_{\max }\right)$, arrives at the warehouse, which declines at a constant rate.

2. There are $\mathrm{f}$ units of raw-materials need to produce a finished product as well as during manufacturing run time $T_{\text {run }}$ where $T_{\text {run }}=\frac{D T}{P}$, raw-material is declined at the rate of $p$. 
3. During the Production, defective items are produced. After first stage inspection, $\alpha$ percentage of defective items is occurred. Then we have $(1-\alpha) Q_{0}$ number of perfect items.

4. Supplier delivered merely perfect item to the buyer with a little quantity $q,(q \leq Q)$. The perfect products have been shifted to the buyer with a fixed period $\frac{q}{D},(D \leq q)$.

5. Next the defective items will send for rework. If the rework was completed then the second inspection occurs. At the second stage, we have $\beta$ proportion good products of the imperfect products. At the end of second stage inspection dispose the defective items. In this time, $\beta \alpha Q_{0}$ perfect items are obtained and $(1-\beta) \alpha Q_{0}$ imperfect products are disposed. No reserves stock and Shortage occurred here.

\section{MATHEMATICAL MODEL}

\subsection{Inventory model for the vendor}

Similar to Fauza et al. [7], this study adopts the kinetic model approach to symbolize the quality loss rate of rawmaterial as expressed in Eq. (1)

$$
\frac{d Q_{R}}{d t}=\varphi Q_{R}^{\eta}
$$

where $\mathrm{Q}_{\mathrm{R}}$ symbolizes the quality recital like gluten content, $t$ and $k$ indicate time and deterioration rate respectively, whereas $\eta$ states the power factor or the reaction order.

The quality of raw material is considered to be at its maximum value $\left(Q_{R}^{\max }\right)$ when it enters the vendor's warehouse and then its quality declines over time following Eq. (1). Also considering that the raw-material has a constant declining grate $(\eta=0)$, therefore the quality state over time can be mathematically denoted by $Q_{R}(t)=$ $Q_{R}^{\max }-k t$. Raw-material arrives at the vendor's warehouse at the beginning of each cycle production run time $\mathrm{T}_{\text {run }}=$ $\frac{D T}{P}$. Hence, it arrives at the manufacturer's warehouse in the intervals of $\frac{T_{\text {run }}}{m}$ that is $\frac{D T}{m P}$ and its quality starts degrading during the cycle time at rate $\mathrm{k}$.

As mentioned earlier, for the raw-material consumed for production at time $t$, where $t$ is the time indicating the duration of this raw material stored in the warehouse, it will incur a quality lost by $Q_{R}^{\max }-Q_{R}(t)$ with a cost of $c_{\text {loss }}$.

Thus, the total cost of quality loss per unit time $\mathrm{f}(m, \mathrm{~T})$ of the raw-material for all batches over one production cycle time $T$ can be expressed by $f(m, T)=\frac{c_{\text {loss }} m P}{T} \int_{0}^{\frac{D T}{m P}} k t d t=C_{\text {loss }} k \frac{D^{2} T}{2 m P}$.

The expected joint total cost for the raw material per unit time is the sum of purchase cost, delivery cost, inventory holding cost and quality loss cost is $p_{\text {raw }} D+z_{\text {raw }} \frac{m}{T}+\left(H_{\text {raw }}+C_{\text {loss }} k\right) \frac{D^{2} T}{2 m P}$.

Suppose an order of $\mathrm{Q}_{0}$ quantity is received from the buyer to the manufacturer, then the manufacturer begins the production at a rate $\mathrm{p}_{0}$ of lot size quantity $\mathrm{Q}_{0}$. To produce the quantity $\mathrm{Q}_{0}$, the mechanism has to retain long-run production process, in which the production of imperfect products may appear.

We consider that $\alpha$ proportion of production rate of imperfect items. Hence $\alpha \mathrm{Q}_{0}$ quantities are defective products, which are sent to vendor for reworking and rest quantities $(1-\alpha) Q_{0}$ are perfect product. After rework $\beta$ proportion of perfect items are found within on the rework products $\alpha \mathrm{Q}_{0}$ and get the perfect quantities $\beta \alpha \mathrm{Q}_{0}$. Remaining $(1-\beta) \alpha Q_{0}$ defective products are disposed. Hence, the total number of perfect products in the production system is $Q=(1-\alpha+\alpha \beta) Q_{0}=u Q_{0}$ which are delivered to the buyer, where $u=1-\alpha+\alpha \beta$.

We consider that the buyer does not claim any kind of demand for the shortage amount $\left(Q_{0}-Q\right)$. Similarly the production the production rate of perfect item is $P=u P_{0}$.

On the production and inspection time, the manufacturer deliverers perfect products to buyer at a small quantity $\mathrm{q}(q \leq Q)$ with a fixed period $\frac{q}{D}$, where $\mathrm{D}(D \leq q)$ is the demand rate of buyer.

There are no reserved stocks to meet the instant demand. The replenishment cycle period is $\left[0, \frac{Q}{D}\right]$ and cycle time $T=\frac{Q}{D}$ has two parts, one $t_{1}=\left[0, \frac{Q}{P}\right]$ and another $t_{1}=\left[\frac{Q}{P}, \frac{Q}{D}\right]$.

In time $t$, the inventory level at the production is the excess of the total production $Q_{P}(t)$ over the delivery $Q_{d}(t)$, where $0 \leq t \leq \frac{Q}{D}$. At time $\mathrm{t}$, during the replenishment cycle, the total production $Q_{P}(t)$ can be represented by 


$$
Q_{P}(t)= \begin{cases}P_{0} t, & 0 \leq t \leq \frac{Q}{P}, \\ Q, & \frac{Q}{P} \leq t \leq \frac{Q}{D}\end{cases}
$$

Therefore, the total production over the cycle time $\mathrm{T}$ is $\int_{0}^{\frac{Q}{D}} Q_{P}(t) d t=\left(\frac{1}{D}-\frac{2 u-1}{2 P u}\right) Q^{2}$

Here the initial delivery quantity $q$ is delivered at time $\frac{Q}{D}$ and equally shipped $n$ times.

Hence total delivery quantity on the replenishment cycle $\mathrm{T}$ is $\int_{0}^{T=\frac{Q}{D}} Q_{d}(t) d t=\int_{0}^{\frac{n q}{D}} Q_{d}(t) d t=\frac{n^{2} q^{2}}{2 D}$.

Hence the total on-hand inventory is the difference between total production and total delivery.

That is $\left(\frac{1}{D}-\frac{2 u-1}{2 P u}\right) Q^{2}-\frac{n^{2} q^{2}}{2 D}$.

Thus the total on-hand inventory per unit time is $\left[1-\frac{(2 u-1) D}{2 P u}\right] Q-\frac{n q}{2}$.

Let $g_{0}$ indicate fuel consumption of the empty vehicle and $g_{1}$ indicate unit fuel consumption factor if the vendor's transportation vehicle is loaded with goods. This research considers $g_{1} Q$ captures amount of fuel consumption for a one-way delivery from vendor to buyer. We consider that backhaul is not used and return vehicles are unloaded, and that $g_{0}$ is amount of the fuel consumption for the return journey. Thus, for one inventory replenishment, the total amount of fuel consumption is $g_{0}+g_{1} Q$. So vendor's mean transportation cost per unit of time is $\frac{c D}{Q}\left(g_{0}+g_{1} Q\right)$.

In this system, we compute the total amount of carbon emissions from transportation and storage. Thus the related amount of carbon emissions is designed by

$$
\Pi(Q, n)=\Pi_{1}(Q, n)+\Pi_{2}(Q, n)=n \theta_{1}\left(g_{0}+g_{1} Q\right)+\left(w_{0}+w_{1} E H I(Q, P, n)\right) \theta_{2}
$$

Here $\Pi_{1}(Q, n)$ and $\Pi_{2}(Q, n)$ are the amounts of carbon emissions from transportation and storage, respectively. Then $\theta_{1}$ and $\theta_{2}$ are carbon emission factors for fuel and electricity. In $\Pi_{2}(Q, n), w_{0}$ and $w_{1}$ are fixed and unit variable electricity consumption for storage. Accordingly, the vendor's carbon emissions cost for all products per unit time is obtained by

$$
\Pi(Q, n)=\frac{D c_{e} n \theta_{1}}{Q}\left(g_{0}+g_{1} Q\right)+\left(w_{0}+w_{1}\left[1-\frac{(2 u-1) D}{2 P u}\right] Q-\frac{n q}{2}\right) c_{e} \theta_{2} .
$$

Hence the total cost for the manufactured product is the sum of holding cost, set up cost, inspection cost, rework cost and disposal cost. That is

$$
H_{1}\left[1-\frac{(2 u-1) D}{2 P u}\right] n q-H_{1} \frac{n q}{2}+\frac{S D}{n q}+\frac{D}{u}\left(C_{0}+C_{1} \alpha+C_{0} \alpha+C_{2}(1-\beta) \alpha\right)
$$

Thus total cost function for the vendor per unit time is sum of raw material cost, manufactured cost , transportation cost and carbon emission cost. That is

$$
\begin{aligned}
\operatorname{TCV}(n, q)=p_{\text {raw }} D & +\emptyset_{\text {raw }} \frac{m D}{n q}+\left(H_{\text {raw }}+C_{\text {loss }} k\right) \frac{D n q}{2 m P}+H_{1}\left[1-\frac{(2 u-1) D}{2 P u}\right] n q-H_{1} \frac{n q}{2}+\frac{S D}{n q} \\
& +\frac{D}{u}\left(C_{0}+C_{1} \alpha+C_{0} \alpha+C_{2}(1-\beta) \alpha\right)+\frac{c D}{n q}\left(g_{0}+g_{1} n q\right)+\frac{D c_{e} n \theta_{1}}{n q}\left(g_{0}+g_{1} n q\right) \\
& +\left(w_{0}+w_{1}\left[1-\frac{(2 u-1) D}{2 P u}\right] n q-\frac{n q}{2}\right) c_{e} \theta_{2}
\end{aligned}
$$

\subsection{Total cost for Buyer}

For the buyer, there are $n$ fixed handling cost to process the $n$ delivered shipments, fixed ordering cost and the expected on-hand inventory level is $\mathrm{I}_{2}=\frac{q}{2}$ in a single replenishment cycle $\left(\frac{Q}{D}\right)$.

So the total cost for buyer per unit time is sum of ordering cost, handling cost and holding cost. That is

$$
\operatorname{TCB}(q, n)=\frac{D A_{1}}{n q}+\frac{D H_{3}}{q}+\frac{D H_{2}}{2 n}
$$

Lastly the expected total cost function for the system is sum of total cost of vendor as well as buyer. That is $\operatorname{ETCM}(q, n)=\operatorname{TCV}(q, n)+\operatorname{TCB}(q, n)$. 


$$
\begin{aligned}
\operatorname{ETCM}(n, q)= & p_{\text {raw }} D+z_{\text {raw }} \frac{m D}{n q}+\left(H_{\text {raw }}+C_{\text {loss }} k\right) \frac{D n q}{2 m P}+H_{1}\left[1-\frac{(2 u-1) D}{2 P u}\right] n q-H_{1} \frac{n q}{2}+\frac{S D}{n q} \\
& +\frac{D}{u}\left(C_{0}+C_{1} \alpha+C_{0} \alpha+C_{2}(1-\beta) \alpha\right)+\frac{c D}{n q}\left(g_{0}+g_{1} n q\right)+\frac{D c_{e} n \theta_{1}}{n q}\left(g_{0}+g_{1} n q\right) \\
& +\left(w_{0}+w_{1}\left[1-\frac{(2 u-1) D}{2 P u}\right] n q-\frac{n q}{2}\right) c_{e} \theta_{2}+\frac{D A_{1}}{n q}+\frac{D H_{3}}{q}+\frac{D H_{2}}{2 n}
\end{aligned}
$$

\section{SOLUTION PROCEDURE}

Proposition1 For fixed $q$, ETCM $(n, q)$ is convex in $n$.

Proof.

Taking first and second derivative of (4) with respect to q, we have

$\frac{\partial \operatorname{ETCM}(n, q)}{\partial n}=-Z_{\text {raw }} \frac{m D}{n^{2} q}+\left(H_{\text {raw }}+C_{\text {loss }} k\right) \frac{D q}{2 m P}+H_{1}\left[1-\frac{(2 u-1) D}{2 P u}\right] q-H_{1} \frac{q}{2}-\frac{S D}{n^{2} q}-\frac{c D}{n^{2} q} g_{0}+D c_{e} \theta_{1} g_{1}+$ $c_{e}\left(w_{1}\left[1-\frac{(2 u-1) D}{2 P u}\right] q-\frac{q}{2}\right) \theta_{2}-\frac{D A_{1}}{n^{2} q}-\frac{D H_{2}}{2 n^{2}}$.

and $\frac{\partial^{2} \operatorname{ETCM}(n, q)}{\partial n^{2}}=\frac{2 D}{n^{3 q}}\left(S+c g_{0}+z_{\text {raw }} m+A_{1}+\frac{H_{2} q}{2}\right)>0$.

Therefore for fixed $q, \operatorname{ETCM}(n, q)$ is convex in $\mathrm{n}$.

This completes the proof of Proposition 1.

Result 1. We get the subsequent optimal number of shipment in the whole planning horizon for the fixed value of $q$ by setting Eq. (5) equal to zero.

$$
n=\sqrt{\frac{D\left[z_{\text {raw }} m+S+C g_{0}+A_{1}\left(\frac{q H_{2}}{2}\right)\right]}{q\left[\left(H_{\text {raw }}+C_{\text {loss }} k\right) \frac{D q}{m P}+H_{1} q\left(\frac{1}{2}-\frac{(2 u-1)}{2 P u} D\right)+D c_{e} g_{1} \theta_{1}+c_{e}\left(w_{1}\left\{1-\frac{(2 u-1) D}{2 P u}\right\} q-\frac{q}{2}\right) \theta_{2}\right]}}
$$

Proposition 2. For fixed $n, \operatorname{ETCM}(n, q)$ is convex in $q$.

Proof.

Taking first and second derivative of (4) with respect to q, we have

$\frac{\partial \operatorname{ETCM}(n, q)}{\partial q}=-Z_{\text {raw }} \frac{m D}{n q^{2}}+\left(H_{\text {raw }}+C_{\text {loss }} k\right) \frac{D n}{2 m P}+H_{1}\left[1-\frac{(2 u-1) D}{2 P u}\right] n-H_{1} \frac{n}{2}-\frac{S D}{n q^{2}}-\frac{D g_{0}}{q^{2}}\left[\frac{c}{n}+c_{e} \theta_{1}\right]+$

$c_{e}\left[w_{1}\left(1-\frac{(2 u-1) D}{2 P u}\right) n-\frac{n}{2}\right] \theta_{2}-\frac{D}{q^{2}}\left[\frac{A_{1}}{n}+H_{3}\right]$.

$\frac{\partial^{2} \operatorname{ETCM}(n, q)}{\partial q^{2}}=2 z_{\text {raw }} \frac{m D}{n q^{3}}+\frac{2 S D}{n q^{3}}+\frac{2 D g_{0}}{q^{3}}\left[\frac{c}{n}+c_{e} \theta_{1}\right]+\frac{2 D}{q^{3}}\left[\frac{A_{1}}{n}+H_{3}\right]>0$.

Therefore for fixed $n, \operatorname{ETCM}(n, q)$ is convex in q.

This completes the proof of Proposition 2.

Result 2. We get the subsequent optimal delivery quantity of perfect goods for buyer for the fixed value of $n$ by setting Eq. (6) equal to zero.

$$
q=\sqrt{\frac{2 D\left[z_{\text {raw }} m+S+g_{0}\left(C+n c_{e} \theta_{1}\right)+A_{1}+n H_{3}\right]}{n^{2}\left[\left(H_{\text {raw }}+C_{\text {loss }} k\right) \frac{D}{m P}+H_{1}\left(1-\frac{(2 u-1) D}{2 P u}\right)+c_{e}\left(w_{1}\left\{2-\frac{(2 u-1) D}{P u}\right\}-1\right) \theta_{2}\right]}}
$$

\section{NUMERICAL EXAMPLE}

Consider the two stage inspection inventory system with the following data: $\mathrm{D}=1600$ units/year, $\mathrm{P}=2500$ units/year, $\mathrm{S}=\$ 250 / \mathrm{setup}, A_{1}=\$ 110$ /order, $p_{\text {raw }}=\$ 7 / \mathrm{unit}, z_{\text {raw }}=\$ 4 / \mathrm{unit}, H_{\text {raw }}=\$ 5 / \mathrm{unit}, C_{\text {loss }}=\$ 4 / \mathrm{unit}$, $\mathrm{m}$ $=10 /$ production cycle,$g_{0}=\$ 15 /$ delivery, $g_{1}=\$ 20 /$ delivery, $\quad \theta_{1}=1, \theta_{2}=0.6, w_{0}=0.3, w_{1}=0.2, c=\$ 20 /$ unit, $c_{e}=$ $\$ 15 /$ unit, $H_{1}=\$ 12 /$ unit, $H_{2}=\$ 11 /$ unit, $H_{3}=\$ 13 /$ unit, $\mathrm{C}_{0}=\$ 2 /$ unit, $\mathrm{C}_{1}=\$ 3 /$ unit, $\mathrm{C}_{2}=\$ 2 /$ unit, $\mathrm{k}=0.01, \alpha=10 \%, \beta=99 \%$ ( since $\mathrm{u}=1-\alpha+\alpha \beta)$. Then we obtain the minimum total expected cost $\operatorname{ETCM}\left(q^{*}, n^{*}\right)$ is $\$ 1356.26$ with the corresponding optimal values $q^{*}=118$ units and $n^{*}=5$. The plot of the total cost versus $n$ is given in Fig. 1 . 


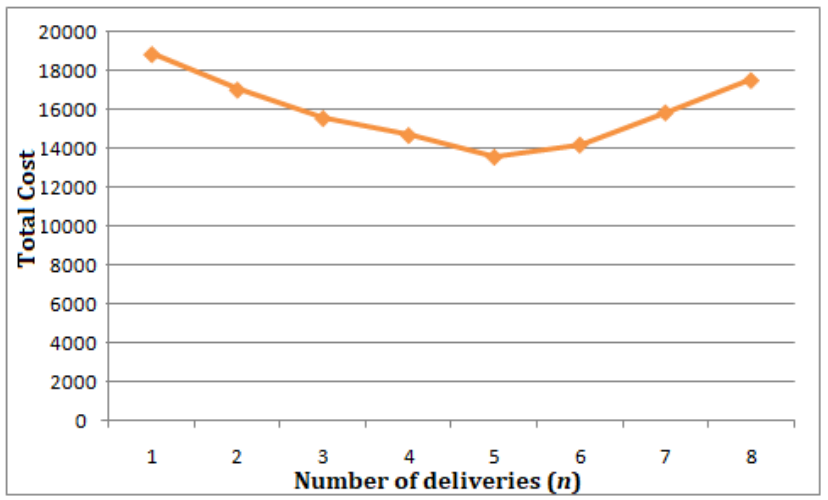

Fig.1. Plot of the total cost versus $n$

\subsection{Sensitivity Analysis}

In this section, the sensitivity analysis is executed. The results are given in Table 1and plots are shown in Figure 2.

Table 1. Sensitivity analysis of various parameters

\begin{tabular}{|c|c|c|c|c|}
\hline Parameters & $\begin{array}{c}\text { Percentage } \\
\text { changes }\end{array}$ & $q^{*}$ & $n^{*}$ & $\operatorname{ETCM}()$. \\
\hline \multirow{5}{*}{$D$} & $+50 \%$ & 287 & 3 & 1895.65 \\
\hline & $+25 \%$ & 185 & 3 & 1652.28 \\
\hline & $0 \%$ & 118 & 5 & 1356.26 \\
\hline & $-25 \%$ & 100 & 5 & 1245.69 \\
\hline & $-50 \%$ & 95 & 6 & 1122.21 \\
\hline \multirow{5}{*}{$P$} & $+50 \%$ & 245 & 5 & 1725.63 \\
\hline & $+25 \%$ & 159 & 5 & 1587.27 \\
\hline & $0 \%$ & 118 & 5 & 1356.26 \\
\hline & $-25 \%$ & 98 & 5 & 1232.12 \\
\hline & $-50 \%$ & 80 & 6 & 1025.36 \\
\hline \multirow{5}{*}{$A$} & $+50 \%$ & 135 & 5 & 1489.87 \\
\hline & $+25 \%$ & 129 & 5 & 1398.47 \\
\hline & $0 \%$ & 118 & 5 & 1356.26 \\
\hline & $-25 \%$ & 102 & 5 & 1289.28 \\
\hline & $-50 \%$ & 95 & 5 & 1198.87 \\
\hline \multirow{5}{*}{$S$} & $+50 \%$ & 140 & 5 & 1587.54 \\
\hline & $+25 \%$ & 128 & 5 & 1474.29 \\
\hline & $0 \%$ & 118 & 5 & 1356.26 \\
\hline & $-25 \%$ & 100 & 5 & 1287.52 \\
\hline & $-50 \%$ & 98 & 5 & 1258.54 \\
\hline
\end{tabular}




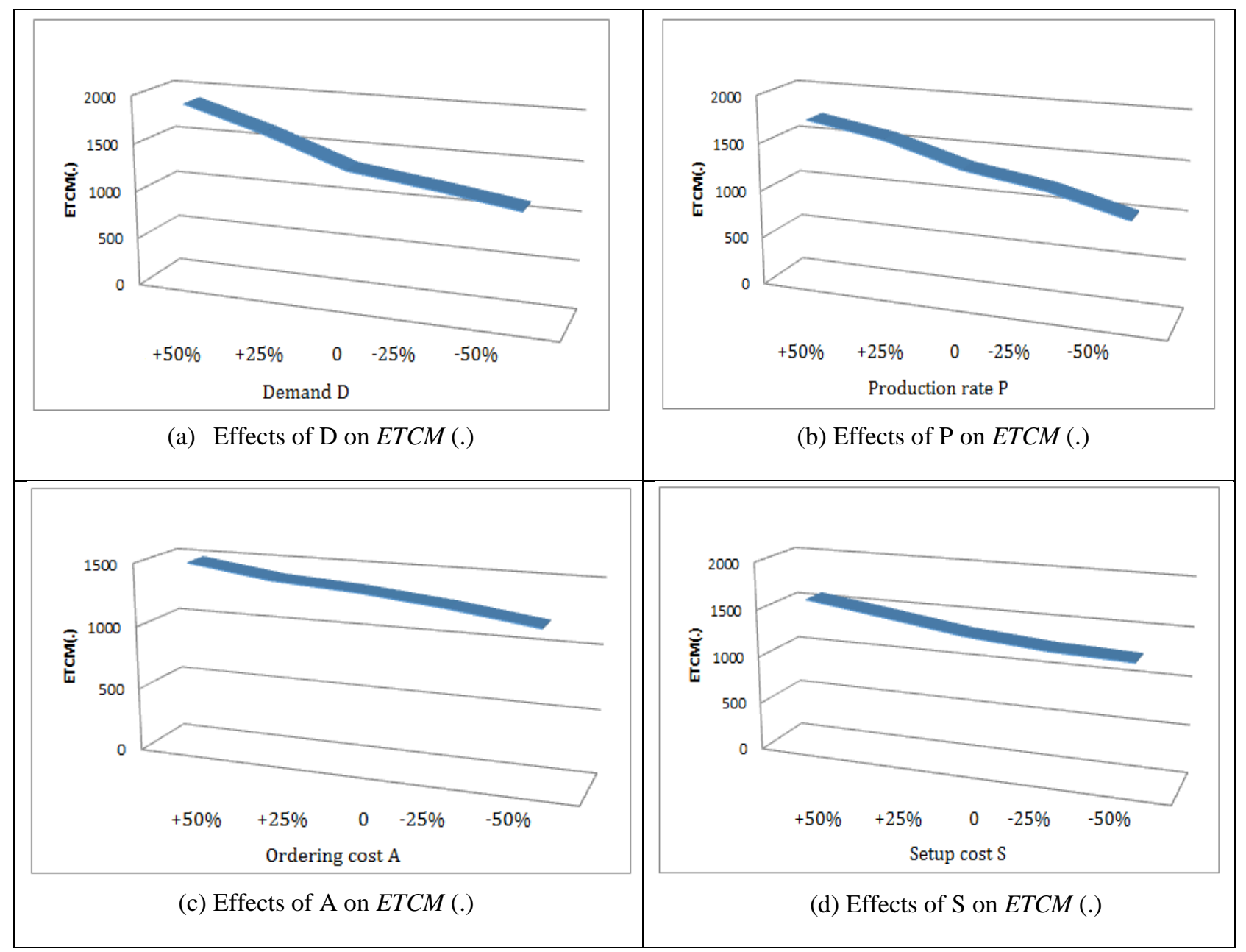

Fig. 2. Plots of sensitivity analysis for various parameters.

\section{CONCLUSION}

In this study, we mathematically derived the two-echelon inventory model for defective products with the reflection of imperfect production process, rework incorporating quality degradation and carbon emission. The research can be used in industries like textile and footwear, chemical, food, etc. An analytical method is employed to optimize the order quantity, total number of shipments and carbon cost for minimum expected joint system cost.

\section{REFERENCES}

[1] S. Priyan, P. Mala, "Optimal inventory strategies for multi-echelon supply chain system involving carbon emissions and fuzzy deterioration”, International Journal of Logistics Systems and Management, vol. 37, no.3, pp. 324-351

[2] Hoque, M.A. "An optimal solution policy to an integrated manufacturer-retailers problem with normal distribution of lead times of delivering equal and unequal-sized batches", OPSEARCH, Article in press.

[3] Chun-JenChung. "Investigating imperfect process and demand effects on inspection scheduling and supply chain replenishment policy”, Computers \& Industrial Engineering, vol. 64, no. 1, pp. 31-44, 2013.

[4] Darwish M.A, Ben-Daya M, "Effect of inspection errors and preventive maintenance on a two stage production inventory system” International Journal of Production Economics, vol.107, no. 1, pp.301-313, 2007.

[5] BennyMantin, LifeiJiang, "Strategic Inventories with Quality Degradation". European Journal of Operational Research, vol. 258, no.1, pp. 155-164, 2017.

[6] S.Priyan, R. Uthayakumar, "Mathematical modeling and computational algorithm to solve multi-echelon multiconstraint inventory problem with errors in quality inspection" Journal of Mathematical Modeling and Algorithms in Operations Research, vol. 14, no. 1, pp. 67-89 
[7] Gusti Fauza et al., "An integrated single-vendor multi-buyer production-inventory policy for food products incorporating quality degradation", International Journal of Production Economics, vol.182, no.1, pp.409-417, 2016.

[8] S. Priyan, P. Mala (2020). Optimal inventory system for pharmaceutical products incorporating quality degradation with expiration date: A game theory approach. Operations Research for Healthcare 24 (1) 100245

[9] Li J. et al., "Production and transportation outsourcing decisions in the supply chain under single and multiple carbon policies", Journal Cleaner and Production, vol. 141, no.1, pp.1109-1122, 2017.

[10] Zhengyi and Jiangtao Hai. "Inventory management for one warehouse multi retailer system with carbon emission cost. Computers and Industrial Engineering, vol. 130, no. 2, pp.565-574, 2019. 